\title{
Genome-wide identification and characterization of barley bHLH transcription factors and their expression in response to low nitrogen stress
}

\section{Xiaoyan Quan}

University of Jinan

Xiaoli Liang

University of Jinan

Chunjuan Xie

University of Jinan

Ning Yin

University of Jinan

Ning Zhang

University of Jinan

Hongmei Li

University of Jinan

Wenxing He ( $\nabla$ 163.hwx@163.com )

University of Jinan

\section{Research Article}

Keywords: Genome-wide analysis, bHLH transcription factor, Barley, Low nitrogen, Expression pattern

Posted Date: December 9th, 2020

DOl: https://doi.org/10.21203/rs.3.rs-117249/v1

License: (9) This work is licensed under a Creative Commons Attribution 4.0 International License. Read Full License 


\section{Abstract}

Background: Improvement of low nitrogen (LN) tolerance or nitrogen use efficiency (NUE) in crops is imperative for environment-friendly agriculture development. The basic helix-loop-helix (bHLH) transcription factors are involved in multiple abiotic stress, suitable as the candidate genes for improving $\mathrm{LN}$ tolerance. Little research was done on characterization of $b H L H$ gene family and their response to $\mathrm{LN}$ stress in barley.

Results: In this study, $168 \mathrm{bHLH}$ genes were identified in barley through genome-wide analysis. HvbHLH proteins were classified into 26 subfamilies based on phylogenetic analysis with bHLH proteins from Arabidopsis thaliana and rice. The analysis of conserved motifs and gene structures supported the evolutionary relationships among these HvbHLH proteins. Further, analysis of stress-related cis-elements in the promoter regions showed that bHLH proteins in barley are probably involved in multiple stress responses. Finally, at least 16 bHLH genes were differentially expressed in two barley genotypes differing in $L N$ tolerance under LN stress. Dynamic expression analysis showed that these differentially expressed genes (DEGs) differed between the two barley genotypes in response to LN stress.

Conclusion: It is the first genome-wide analysis of bHLH family genes in response to LN stress in barley. The results indicate the distinct difference among $H v b H L H$ genes in response to various abiotic stresses. The HvbHLHs specifically expressed in the LN-tolerant barley genotype XZ149 identified herein may be valuable for future function analysis of $H v b H L H$ genes under $L N$ stress and breeding for barley cultivars with LN tolerance.

\section{Introduction}

Transcription factors (TFs) play important roles in the growth and development of plants and animals, and their responses to the external environment via regulating downstream gene expression. As one of the most critical factors regulating gene expression, TFs have been intensively concerned in the field of biological research. Among various TF families, the basic-helix-loop-helix (bHLH) TFs constitute one of the largest TF families [1]. The bHLH proteins are characterized by a conserved bHLH domain consisting of about 60 amino acids. Each domain has two functional regions, i.e. the basic region at $\mathrm{N}$-terminus and the $\mathrm{HLH}$ region at the $\mathrm{C}$-terminus. The basic region comprises about 15 amino acids including six basic residues, and has DNA-binding activity [2]. The HLH region, containing about 50 amino acids, is composed of two a-helices separated by a variable loop, and it participates in the formation of homodimers or heterodimers [3].

The bHLH TFs were first identified and characterized in mammals and then discovered gradually in other eukaryotic species. At present, genome-wide comprehensive analyses of plant bHLH proteins have been carried out in numerous species [4]. At least 162 and $167 \mathrm{bHLHs}$ have been identified in model plants Arabidopsis and rice, respectively [5-7]. Recently, 225, 208, 155, 152, 124 and 142 bHLHs were identified in 
wheat, maize, bean, tomato, potato, and cucumber, respectively [4, 8-12]. However, the similar research has been little done in barley, although barley is an important crop worldwide.

TFs are the excellent candidate genes for developing cultivars with improved tolerance to adverse stress [13-15]. In plants, bHLH TFs are involved in many biological processes, such as the regulation of flavonoid biosynthesis, morphology and the accumulation of fruit pigments [16-17]. Some bHLH TFs participate in regulation of biotic and abiotic stress responses [18-20]. For example, some bHLH TFs confer drought tolerance through regulating stomatal development, photosynthesis and growth [21], or by promoting root development and abscisic acid synthesis [22]. Some enhance plant salt stress [23], cold [24], or manganese [25] through their overexpression. And some could improve plant growth while subjected to nutrition deficiency, such as iron, $\mathrm{Pi}$ and $\mathrm{N}$ deficiency [26-28].

Environmental pollution caused by excessive application of nitrogen $(\mathrm{N})$ fertilizers became a big issue. One of the efficient solutions is to develop cultivars with high $\mathrm{N}$ use efficiency /low nitrogen (LN) tolerance. It is reported that bHLH could improve plant tolerance to $\mathrm{N}$ deficiency [28]. Thus, $b H L H$ genes may be used as suitable candidates for the genetic improvement of plant $L N$ tolerance/high $\mathrm{N}$ use efficiency. However, up to date little has been known about the function of plant bHLH proteins. In this study, $b H L H$ family genes in barley were identified and characterized at genome-wide level. It was found that $H v b H L H$ genes were differentially expressed in the two barley genotypes (XZ149, LN tolerant and XZ56, LN sensitive) differing in LN tolerance [29-30] at transcription level under LN stress by RNA-seq. The major objectives of this study were to determine the characteristics of $H v b H L H$ gene family, to explore the differences of $H v b H L H$ gene expression between the two contrasting genotypes in response to $\mathrm{LN}$ stress, and to identify the $H v b H L H$ genes useful for $\mathrm{LN}$ tolerance breeding.

\section{Results}

\section{Identification of $b H L H$ family genes in barley}

Proteins containing bHLH domain in barley were identified by searching the whole barley genome. Totally $168 \mathrm{bHLH}$ genes were obtained in barley genome after removing the redundant protein sequences (Additional file 1 and 2: Table S1 and S2). By EXPASY analysis, we found the amino acid length of HvbHLH proteins ranged from 36 to 938, accordingly the molecular weight (MW) ranging from 9.63 to $102.01 \mathrm{kDa}$ (Additional file 1: Table S1). The isoelectric point (pl) varied greatly, from 4.63 to 11.9 (Additional file 1: Table S1). While the MW and pl of $11 \mathrm{HvbHLH}$ proteins were not predicted, due to existence of some consecutive undefined amino acids (Additional file 1: Table S1).

\section{Phylogenetic analysis of bHLH proteins}

In order to analyze the evolutionary relationship of the $b H L H$ genes between barley and other species, the unrooted phylogenetic tree was constructed using the full-length amino acid sequences of all bHLH in Arabidopsis, rice and barley by the neighbor-joining (NJ) method. The result showed that $450 \mathrm{bHLH}$ proteins from Arabidopsis, rice and barley were divided into 27 subgroups (Subgroup 1 to 27; Fig. 1) 
based on the classification of bHLH proteins in Arabidopsis and rice [6-7]. Except Subgroup 20, all members of $b H L H$ family in barley could be found in all the other subgroups (Fig. 1). Subgroups 3 and 9 were the smallest, each only having three members in barley, rice and Arabidopsis. Subgroup 27 was the largest, having 20, 16 and 17 members in barley, rice and Arabidopsis, respectively. Another NJ phylogenetic tree was also constructed based on HvbHLH protein sequences, and HvbHLHs were classified into 25 subfamilies (Subfamily A to Y, Fig. 2a). Classification in HvbHLH was generally consistent with those in Arabidopsis and rice phylogenic tree (Fig. 1 and 2a).

\section{Analysis of motifs and exon/intron structures}

Ten conserved motifs containing 11 to 57 amino acids were predicted in HvbHLH proteins through MEME software (Additional file 3: Fig. S1). HvbHLH proteins consist of 1- 5 motifs, varying with different subfamilies (Fig. 2b). However, all of them contained at least of the Motif 1 or 2, and the proteins in the same subfamily exhibited the similar motif composition (Figs. 2a and b). For example, Motifs 2, 3, and 10 were identified in all 6 members of Subfamily K, and Motifs 1, 2, and 7 were identified in 12 of the 15 members of Subfamily X. Furthermore, some motifs are unique to one or more Subfamilies. For instance, Motif 5 was specifically shared by each member in the Subfamily D, and motif 3 was only found in Subfamilies $\mathrm{B}, \mathrm{K}$ and $\mathrm{L}$. This conservation of the motif composition patterns in each subfamily might indicate their similar biological functions in the same subfamily [31].

The alignment of genome and coding sequences, and also exon/intron structure within the coding sequence in bHLH genes was performed using the TBtools [32]. The structure differed greatly among $H v b H L H$ genes, but most members of the same subfamily had the similar exon/intron structure (Figs. 2a and c). For instance, the members in the Subfamily A had the relatively simple structure, possessing only 1-2 exons, while the members in the Subfamily T had 6-8 exons, being much more complex in its structure (Fig. 2c).

\section{Chromosomal distribution and gene duplication of $H v b H L H$ genes}

According to their physical positions, $161 \mathrm{HvHLH}$ genes were nonrandomly mapped on seven barley chromosomes, with most located at the both ends of the chromosomes (Fig. 3). More than one-third of the HvbHLH genes were located on Chr3 (30 members) and Chr5 (26 members), and only 12 genes was located on Chr1 (Fig. 3). In addition, $7 \mathrm{HvbHLHs} \mathrm{could} \mathrm{not} \mathrm{be} \mathrm{conclusively} \mathrm{localized} \mathrm{to} \mathrm{a} \mathrm{chromosome}$ (Fig. 3).

Totally 18 segmental duplication events were identified among HvbHLHs (Fig. 4, Additional file 4: Table S3). Among them, 16 events happened between diverse chromosomes, while only 2 events were detected within a same chromosome. Nine genes were identified as tandem duplication genes (Fig. 4, Additional file 4: Table S3), and the genes located on chromosome 3 showed the highest numbers (7) of tandem duplications, while two genes (HORVU4Hr1G087590 and HORVU4Hr1G087610) were tandem duplicated on Chr4 (Fig. 4). Each pair of duplication genes were derived from the same subfamily (Fig. 1 and 4). 
Obviously, some $H v b H L H$ genes were generated due to gene duplication, and the segmental duplication may play vital roles in the $H v b H L H$ gene family expansion in barley genome.

\section{Stress-related ciselements in the promoter of $H v b H L H$ genes}

To understand the potential regulatory mechanisms of $H v b H L H$ genes, cis-elements were analyzed using 1.5-kb upstream sequences from ATG through PlantCARE. Various cis-elements involved in abiotic stress responses were identified, and mainly they could be divided into two groups. One is elements responsive to hormones, including abscisic acid (ABRE), jasmonic acid (CGTCA and TGACG motif), auxin (AuxRRcore and TGA-element), gibberellins (GARE motif, TATC-box and P-box,), salicylic acid (SARE and TCAelements). The other one is responsive to environmental stress, including low-temperature responsive element (LTR), anaerobic induction element (ARE), MYB binding site involved in drought-inducibility

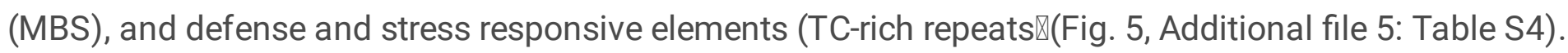
All $H v b H L H$ genes had light responsive cis-elements, belonging to the most commonly predicted ciselements in the promoter of $H v b H L H$ genes, and 155 (92.26\%) HvbHLH genes contained the G-box elements.most genes had more than one kind of cis-elements, suggesting that both groups could respond to multiple stresses.

\section{Expression profiles of $H v b H L H s$ in different plant tissues}

To further understand the functions of $H v b H L H s$, the expression profiles of $H v b H L H$ genes in 8 plant tissues of a barley cultivar 'Morex' were analyzed based on the available transcriptomic data [33]. The expression of $149 \mathrm{HvbHLH}$ genes were identified in all 8 tissues, and their expression levels varied considerably with genes and tissues (Fig. 6 and Additional file 6: Table S5). Some HvbHLHs in the same subfamily showed the similar expression pattern, while some others displayed the different patterns (Fig. 6). Totally 128 (128/149, 85.91\%), 125 (125/149, 83.89\%), 118 (118/149, 79.19\%), and 101 (101/149, 67.79\%) $H v b H L H$ genes expressed in four-day-old embryos, roots and shoots, and internodes of six-leafold seedlings, respectively (Fig. 6). In addition, 118 (118/149, 79.19\%) and 110 (110/149, 67.79\%) $\mathrm{HvbHLH}$ genes expressed in young fluoresce $(5 \mathrm{~mm}$ and $1-1.5 \mathrm{~cm}$ ) and developing grains (5- and 15-day post anthesis, DPA), respectively (Fig. 6).

Some HvbHLHs highly expressed in the specific tissue(s). For instance, the genes HORVU2Hr1G044230 and HORVU6Hr1G020520 showed higher expression in caryopsis (5 and 15 DPA) than other tissues (Fig. 6), suggesting their significant roles in grain growth and development. HORVU2Hr1G044230 and HORVU1Hr1G072810 had higher expression in embryos and roots, while HORVU2Hr1G044230, HORVU7Hr1G050530 and HORVU1Hr1G054260 had higher expression in shoots (Fig. 6), indicating these genes may participate in growth and development of vegetative organs. Notably, HORVU2Hr1G044230 displayed extremely high expression in all the tissues (Fig. 6).

\section{Expression profiles of $H v b H L H s$ under low nitrogen stress}


The expression patterns of $H v b H L H$ genes in response to $L N$ stress were analyzed in the roots of the two barley genotypes (XZ149, LN tolerant and XZ56, LN sensitive) using RNA-seq (Additional files 7 and 8: Tables S6 and S7). Consequently, 16 differentially expressed genes (DEGs) encoding HvbHLH proteins were identified using pair-wise comparison for each genotype under LN stress (Fig. 7, Additional file 9: Table S8). Remarkably, no DEG was found in the two barley genotypes under LN stress at $12 \mathrm{~d}$, suggesting $H v b H L H s$ may play a regulating role at early stress. Furthermore, all the DEGs were changed only at one time point, except MLOC_74557 (HORVU6Hr1G064820) and MLOC_21066 (HORVU3Hr1G000150), which was down-regulated in XZ149 both at $6 \mathrm{~h}$ and $48 \mathrm{~h}$ after stress (Fig. 7).

Eleven DEGs were down-regulated in their expression under LN stress, and their response patterns showed the marked difference. Six DEGs responded quickly to LN stress (at $6 \mathrm{~h}$ ), and MLOC_37666 (HORVU2Hr1G108480), MLOC_72940 (HORVU2Hr1G117820), MLOC_21066 (HORVU3Hr1G000150) were down-regulated only in XZ149, while MLOC_67937 (HORVU5Hr1G002090) was down-regulated only in XZ56 (Fig. 7). The other five DEGs were changed at $48 \mathrm{~h}$ after LN stress, and among them MLOC_12832 (HORVU5Hr1G066530), MLOC_66385 (HORVU4Hr1G061760) were down-regulated only in XZ149, MLOC_64390 (HORVU3Hr1G027630) only in XZ56, while MLOC_62844 (HORVU2Hr1G066100) and MLOC_43080 (HORVU3Hr1G000150) in both XZ149 and XZ56 (Fig. 7).

Four DEGs were up-regulated under LN stress, and three of them responded at $6 \mathrm{~h}$, with MLOC_72280 (HORVU2Hr1G114070) only in XZ149, and MLOC_36351 (HORVU3Hr1G108680) and MLOC_76486 (HORVU5Hr1G018100) in both XZ149 and XZ56. MLOC_55964 (HORVU4Hr1G075250) was up-regulated at $48 \mathrm{~h}$ in both XZ149 and XZ6(Fig. 7). Notably, the change fold of all the up-regulated DEGs was larger in the tolerant genotype XZ149 relative to the sensitive genotype XZ56 (Fig. 7).

To confirm the transcriptomic results and understand the expression patterns of $H v b H L H s$, the dynamic response to $L N$ stress were examined using real time PCR for the 6 identified DEGs at 8 time points after $\mathrm{LN}$ stress. The expression patterns of $H v b H L H s$ at $6 \mathrm{~h}, 48 \mathrm{~h}$ and $12 \mathrm{~d}$ after $\mathrm{LN}$ stress were highly consistent with those obtained by the transcriptomic analysis. (Additional file 10: Fig. S2). Under LN stress, different $H v b H L H s$ exhibited distinct dynamic expression patterns, suggesting that $H v b H L H s$ may be involved in regulation of $L N$ tolerance in different ways. For instance, MLOC_72280 (HORVU2Hr1G114070) was up-regulated during $1 \mathrm{~h}-2 \mathrm{~d}$ under LN stress, but down-regulated at $3 \mathrm{~d}-12 \mathrm{~d}$ in XZ149, while it was up-regulated at $1 \mathrm{~h}, 3 \mathrm{~h}, 6 \mathrm{~h}$, and $2 \mathrm{~d}$, and down-regulated at $24 \mathrm{~h}$ and $3 \mathrm{~d}-12 \mathrm{~d}$ in XZ56 (Additional file 11: Fig. S3). For MLOC_37666 (HORVU2Hr1G108480), its expression generally showed the decrease with the time of exposed LN stress in XZ149, but increase at $1 \mathrm{~h}, 3 \mathrm{~h}$, and $6 \mathrm{~d}$ under LN stress in XZ56 (Additional file 11: Fig. S3).

\section{Discussion}

The $b H L H T F s$ in higher plants comprise a large family, involving in plant growth, development, and stress responses [2]. It is well documented that $b H L H$ is responsible to many abiotic stresses such as cold, drought and salt stress[18, 34-35], and nutrients such as iron, Pi and $\mathrm{N}$ deficiency $[20,28,36]$. 
Therefore, it is imperative to make the comprehensive analysis of bHLHTFs family. $\mathrm{N}$ deficiency is a common issue in the agricultural production worldwide. Some studies found that bHLHs are involved in LN stress [28], nevertheless, their relationship/ roles of $b H L H$ family genes in $L N$ tolerance has not been clearly defined. In this study, genome-wide identification and characterization of the $b H L H$ gene family in barley were carried out. Furthermore, the expression profiles of $b H L H$ family under $L N$ stress were determined by using two barley genotypes differing largely in $L N$ tolerance.

As a result, totally $168 \mathrm{bHLH}$ genes were identified in barley through genome-wide analysis (Table S1). These $H v b H L H$ genes were distributed over the barley genome, mainly on both ends of the chromosomes (Fig. 3), similar to the distribution of Arabidopsis and rice bHLH genes on their chromosomes [6-7]. Gene duplication is an important event for the evolution of plant genome, leading to the generation of new genes [37-38]. There were at least 14 pairs of duplication genes in barley $b H L H$ family, indicating that gene expansion occurred during the evolution of the barley genome. Moreover, the segmental duplication could be a predominant driving force for the enlargement of $H v b H L H[39]$.

Based on phylogenetic analysis, HvbHLH proteins could be classified into 26 subfamilies (Fig. 1). Motif and gene structure analysis further supported the subfamily division, as members of the same subfamily showed the similar gene structure and motif (Fig. 2), indicating their similar evolutionary origins and biology functions. For instance, in the subfamily K, AtbHLH100 (AT2G41240), AtbHLH101 (AT5G041059), AtbHLH38 (AT3G56970), and AtbHLH39 (AT3G56980) played important roles in regulating iron homeostasis under Fe deficiency in Arabidopsis [19, 26]. In the same subfamily, OsbHLH056/OsIRO2 (Os01g72730) protein is an essential regulator of the genes involved in Fe uptake under Fe-deficient conditions in rice [40]. On the other hand, $H v b H L H s$ displayed diverse expression patterns in plant tissues, and some genes within the same subfamily may have different expression patterns (Fig. 6), indicating that their functions have been differentiated even in the same subfamily or they may perform the same function in the different ways.

A great number of cis-elements associated with abiotic stress responses were discovered in promoter regions of $H v b H L H s$, suggesting that HvbHLHs may be highly associated with the regulation of abiotic stresses response in barley. To further explore the possible functions of the bHLH family in LN tolerance, the expression profiles of $H v b H L H$ genes were analyzed using two barley genotypes differing in $L N$ tolerance. A total of 16 DEGs encoding HvbHLH proteins were identified in the two genotypes after $6 \mathrm{~h}$ and $48 \mathrm{~h}$ treatments (Fig. 7), but no one was found at $12 \mathrm{~d} \mathrm{LN}$ treatment, suggesting that HvbHLHs respond to LN stress only at early time.

There was a large difference in the transcription regulation of $H v b H L H$ between the two barley genotypes. In detail, the change fold of five up-regulated DEGs was much higher in the LN tolerant genotype XZ149 than the LN sensitive genotype XZ56 under LN stress (Fig. 7). In particular, MLOC_72280 (HORVU2Hr1G114070) and MLOC_42946 (HORVU4Hr1G003210) were up-regulated in XZ149, but little changed or down-regulated in XZ56 (Fig. 7). Thus, it is worthy to determine the roles of these HvbHLH genes in XZ149. It has been reported that overexpression of TabHLH1 in tobacco improved plant 
tolerance to $\mathrm{N}$ deprivation via regulation of nitrate transporter (NRT) gene transcription [28]. In Arabidopsis, the expression of specific bHLH transcription factors was enhanced in response to $\mathrm{N}$ deficiency, accompanied by anthocyanin accumulation [41-42]. Thus, we may focus the candidate genes on the roles in anthocyanin synthesis pathway and regulation of NRT gene transcription under LN stress in the further study.

\section{Conclusion}

In summary, 168 bHLH genes were identified in barley by a genome-wide analysis, and their evolutionary relationships were clarified using phylogenetic, conserved motif, and exon/intron structures analyses. The expression profiles revealed that at least $16 \mathrm{HvbHLH}$ genes may mediate LN stress in barley. The specifically expressed $b H L H$ genes in XZ149 may be valuable for further functional characterizations of $b H L H$ genes under $L N$ stress and breeding of barley cultivars with LN tolerance.

\section{Materials And Methods}

\section{Identification of bHLH genes in barley}

The protein sequences of bHLH in Arabidopsis thaliana and Oryza sativa were retrieved from TAIR (http://www.arabidopsis.org) and RGAP (http://rice.plantbiology.msu.edu/), respectively. Barley sequence data were download from IPK (https://webblast.ipk-gatersleben.de/barley_ibsc/) and Gramene (http://ensembl.gramene.org/Hordeum_vulgare/Info/Index). Totally 282 Arabidopsis and rice bHLH protein sequences (list in Additional file 12: Table S9) were used to search against the barley protein sequences using BlastP with a threshold of an e-value $<1 \mathrm{e}^{-10}$ [43-44]. Moreover, the predicted HvbHLH proteins were also searched against the barley protein database employing HMMER program with Hidden Markov Model (HMM) profiles of the bHLH domain (PF00010), obtained from PFAM database (http://pfam.xfam.org/) [45]. After removing redundant protein sequences, the confirmation of the predicted bHLH proteins were performed through NCBI Conserved Domain Search (http://www.ncbi.nlm.nih.gov/Structure/cdd/wrpsb.cgi) and the Pfam (http://pfam.sanger.ac.uk/search) [46-47]. The biophysical properties of bHLH proteins were calculated using the ExPASy (https://web.expasy.org/protparam/) [48].

\section{Phylogenetic analysis}

Multiple alignment was performed based on the sequences of Arabidopsis thaliana, rice and barley using ClustralW (http://www.genome.jp/tools/clustalw/) [49]. The unrooted phylogenetic tree was constructed by MEGA 6.0 software employing the neighbor-joining $(\mathrm{NJ})$ method, bootstrapping with 1000 replicates.

\section{Exon/intron structure and motif analysis}

The exon/intron structure of the barley $b H L H$ genes were determined by alignment of the cDNAs and their corresponding genomic DNA sequences from the barley genome database. The conserved motifs were 
predicted on the online MEME tool (http://meme-suite.org /tools/ meme) [50]). And a diagrammatic sketch structure and the motif composition of $H v b H L H s$ was mapped through TBtools software [32].

\section{Chromosomal location and gene duplication}

The distribution of $H v b H L H$ transcription factors on chromosomes were mapped by MapGene2Chrom online software (http://mg2c.iask.in/mg2c_v2.1/) [51], according to the specific positions of barley genes obtained from IPK (https://webblast.ipk-gatersleben.de/barley_ibsc/). To identify duplication gene, all barley genome sequences were aligned using BLASTP with an e-value of $1 \mathrm{e}^{-10}$, and then segmental and tandem duplications were identified by MCScanX with default [52]. The synteny relationship of HvbHLHs were displayed with circos (http://circos.ca/) [53].

\section{Ciselements in promoter regions of HvbHLHs}

To predict the cis-elements in the promoter regions of $H v b H L H s$, the 1500 bp upstream sequences from the start codon of each $\mathrm{HvbHLH}$ were retrieved. The cis-element distribution was then investigated in PlantCARE (http://bioinformatics.psb.ugent.be/webtools/plantcare/html/) [54].

\section{Plant materials and stress treatments}

Two Tibetan wild barley genotypes XZ149 (LN tolerant) and XZ56 (LN sensitive) were used for transcriptome and real time PCR. The barley seedlings were cultivated with hydroponics in a plant growth chamber $\left(22 / 18{ }^{\circ} \mathrm{C}\right.$, day/night) according to Quan et al. (2016) [55]. Then, three-leaf-stage seedlings were exposed to $0.2 \mathrm{mM} \mathrm{N}$ (LN stress) and $2 \mathrm{mM} \mathrm{N}$ (control).

\section{Expression patterns analysis of $H v b H L H$ genes}

Transcriptomic expression level of $H v b H L H$ genes in 8 different tissues (4-day embryos dissected from germinating grains, roots and shoots from seedling ( $10 \mathrm{~cm}$ shoot stage), developing inflorescence ( $5 \mathrm{~mm}$ and $1-1.5 \mathrm{~cm}$ ), the third internode at six leaves visible stage, 5 and 15 days post-anthesis developing grain (bracts removed)) of the cultivar 'Morex' were obtained from EMBL-EBI (https://www.ebi.ac.uk/gxa/plant/experiments) [33].

The roots in XZ149 and XZ56 were taken for transcriptomic analysis at $6 \mathrm{~h}, 48 \mathrm{~h}$ and $12 \mathrm{~d}$ after LN stress. The threshold for screening DEGs was set as FDR $<0.05$ and FPKM $\geq 1$ at least in one of the samples [56]. Differential expression analysis of HvbHLHs was displayed using FPKM (fragments per kilobase of exon per million fragments mapped reads) [57] and the expression levels are showed as the heatmaps created by TBtools software by a color gradient from low (blue) to high (red) [32].

To confirm the RNA-Seq results and to further explore the expression patterns of $H v b H L H$ genes, the expression of $H v b H L H$ genes in roots was analyzed at 1 h, $3 \mathrm{~h}, 6 \mathrm{~h}, 24 \mathrm{~h}, 48 \mathrm{~h}, 6 \mathrm{~d}$ and $12 \mathrm{~d}$ under LN stress by real time PCR. RNA extraction and the first strand CDNA synthesis were used FastPure Plant Total RNA Isolation Kit and Hiscript III Reverse Transcriptase (Vazyme, China), respectively. The specific- 
gene primers for $H v b H L H$ and internal control gene $H v G A P D H$ were presented in Additional file 13: Table S10. The real time PCR was analyzed on a CFX96 system (USA) with three biological replicates. The relative expression was set as the fold change referred to the expression under control and calculated by the comparative CT method [58].

\section{Abbreviations}

bHLH, basic helix-loop-helix; DEG, differentially expressed gene; DPA, day post anthesis; FPKM, fragments per kilobase of exon per million fragments mapped reads; LN, low nitrogen; N, nitrogen; NRT, nitrate transporter; NUE, nitrogen use efficiency; TFs, transcription factors

\section{Declarations}

\section{Availability of data and materials}

The datasets used and/or analyzed during the current study are available from the first author on reasonable request, and her email address is bio_quanxy@ujn.edu.cn.

\section{Acknowledgments}

Not applicable.

\section{Funding}

This work was supported by the by National Natural Science Foundation of China (31901437 and 31872415) and Scientific Research Leaders Studio of Jinan (2019GXRC052).

\section{Author information}

\section{Affiliations}

School of Biological Science and Technology, University of Jinan, Jinan, 250022, People's Republic of China.

\section{Contributions}

$\mathrm{XQ}$ and $\mathrm{WH}$ designed the research. $\mathrm{XQ}, \mathrm{XL}, \mathrm{CX}, \mathrm{NY}, \mathrm{NZ}$ and $\mathrm{HL}$ performed research. $\mathrm{XQ}$ analyzed data and wrote the manuscript. WH revised the manuscript. All authors have read, edited and approved the current version of the manuscript.

\section{Corresponding author}

Correspondence to Wenxing He. 


\section{Ethics approval and consent to participate}

Not applicable.

\section{Consent for publication}

Not applicable.

\section{Competing interests}

The authors declare that they have no competing interests.

\section{References}

1. Carretero-Paulet L, Galstyan A, Roig-Villanova I, Martinez-Garcia JF, Bilbao-Castro JR, Robertson DL. Genome-wide classification and evolutionary analysis of the bHLH family of transcription factors in Arabidopsis, poplar, rice, moss, and algae. Plant Physiol. 2010; 153:1398-1412.

2. Heim MA, Jakoby M, Werber M, Martin C, Weisshaar B, Bailey PC. The basic helix-loop-helix transcription factor family in plants: a genome-wide study of protein structure and functional diversity. Mol Biol Evol. 2003; 20:735-47.

3. Massari ME, Murre C. Helix-loop-helix proteins: regulators of transcription in eucaryotic organisms. Mol Cell Biol. 2000; 20:429-40.

4. Kavas M, Baloglu MC, Atabay ES, Ziplar UT, Dasgan HY, Ünver T. Genome-wide characterization and expression analysis of common bean bHLH transcription factors in response to excess salt concentration. Mol Genet Genomics. 2016; 291:129-143.

5. Bailey PC, Martin C, Toledo-Ortiz G, Quail PH, Huq E, Heim MA, Jakoby M, Werber M, Weisshaar B. Update on the basic helix-loop-helix transcription factor gene family in Arabidopsis thaliana. Plant Cell. 2003; 15:2497-2502.

6. Li, X, Duan, X, Jiang, H, Sun, Y, Tang, Y, Yuan, Z, Guo J, Liang W, Chen L, Yin J, Ma H, Wang J, Zhang, D. Genome-wide analysis of basic/helix-loop-helix transcription factor family in rice and Arabidopsis. Plant Physiol. 2006; 141:1167-1184.

7. Toledo-Ortiz G, Huq E, Quail P H. The Arabidopsis Basic/Helix-Loop-Helix Transcription Factor Family. Plant Cell. 2003; 15:1749-1770.

8. Guo XJ, Wang JR. Global identification, structural analysis and expression characterization of bHLH transcription factors in wheat. BMC Plant Biol. 2017; 17: 90.

9. Zhang T, Lv W, Zhang, H., Ma, L., Li, P., Ge, L., Li, G. (2018). Genome-wide analysis of the basic HelixLoop-Helix (bHLH) transcription factor family in maize. BMC Plant Biol. 2018; 18:235.

10. Wang J, Hu Z, Zhao T, Yang Y, Chen T, Yang M, Yu W, Zhang B. Genome-wide analysis of bHLH transcription factor and involvement in the infection by yellow leaf curl virus in tomato (Solanum lycopersicum). BMC Genomics. 2015; 16: 39. 
11. Wang R, Zhao P, Kong N, Lu R, Pei Y, Huang C, Ma H, Chen Q. 2018. Genome-wide identification and characterization of the potato bHLH transcription factor family. Genes. 2018, 9:54.

12. Li J, Wang T, Han J. Ren Z. Genome-wide identification and characterization of cucumber bHLH family genes and the functional characterization of $\mathrm{CsbHLH041}$ in $\mathrm{NaCl}$ and ABA tolerance in Arabidopsis and cucumber. BMC Plant Biol. 2020; 20:272.

13. Thomashow MF, Gilmour SJ, Stockinger EJ, Jaglo-Ottosen KR, Zarka DG. Role of the Arabidopsis CBF transcriptional activators in cold acclimation. Physiol. Plant. 2001; 112:171-175.

14. Nakashima K, Ito Y, Yamaguchi-Shinozaki K. Transcriptional regulatory networks in response to abiotic stresses in Arabidopsis and grasses. Plant Physiol. 2009; 149:88-95.

15. Golldack D, Lüking I, Yang O. Plant tolerance to drought and salinity: stress regulating transcription factors and their functional significance in the cellular transcriptional network. Plant Cell Rep. 2011; 30:1383-1391.

16. Xu W, Dubos C, Lepiniec L. Transcriptional control of flavonoid biosynthesis by MYB-bHLH-WDR complexes. Trends Plant Sci. 2015; 20:176-185

17. Zhu Z, Chen G, Guo X, Yin W, Yu X, Hu J, Hu Z. Overexpression of SIPRE2, an atypical bHLH transcription factor, affects plant morphology and fruit pigment accumulation in tomato. Sci Rep. $2017 ; 7: 5786$

18. Mao K, Dong Q, Li C, Liu C, Ma F. Genome wide identification and characterization of apple bHLH transcription factors and expression analysis in response to drought and salt stress. Front Plant Sci. $2017 ; 8: 480$.

19. Liang G, Zhang H, Li X, Ai Q, Yu D. bHLH transcription factor bHLH115 regulates iron homeostasis in Arabidopsis thaliana. J Exp Bot. 2017; 68:1743-1755.

20. Long TA, Tsukagoshi H, Busch W, Lahner B, Salt DE, Benfey PN. The bHLH transcription factor POPEYE regulates response to iron deficiency in Arabidopsis roots. Plant Cell. 2010; 22:2219-2236.

21. Dong Y, Wang C P, Han X, Tang S, Liu S, Xia X, Yin W. A novel bHLH transcription factor PebHLH35 from Populus euphratica confers drought tolerance through regulating stomatal development, photosynthesis and growth in Arabidopsis. Biochem Bioph Res Co. 2014; 450:453-458.

22. Li Z, Liu C, Zhang Y, Wang B, Ran Q, Zhang J. The bHLH family member ZmPTF1 regulates drought tolerance in maize by promoting root development and abscisic acid synthesis. J Exp Bot. 2019; 70:5471-5486.

23. Zheng K, Wang Y, Wang S. The non-DNA binding bHLH transcription factor Paclobutrazol resistances are involved in the regulation of ABA and salt responses in Arabidopsis. Plant Physiol Bioch. 2019, 139:239-245.

24. Geng J, Wei T, Wang Y, Huang X, Liu JH. Overexpression of PtrbHLH, a basic helix-loop-helix transcription factor from Poncirus trifoliata, confers enhanced cold tolerance in pummelo (Citrus grandis) by modulation of $\mathrm{H}_{2} \mathrm{O}_{2}$ level via regulating a CAT gene. Tree Physiol. 2019; 39:2045-2054. 
25. Sun $\mathrm{K}$, Wang $\mathrm{H}$, Xia Z. The maize bHLH transcription factor bHLH105 confers manganese tolerance in transgenic tobacco. Plants Sci. 2019; 280:97-109.

26. Li X, Zhang H, Ai Q, Liang G, Yu D. Two bHLH transcription factors, bHLH34 and bHLH104, regulate iron homeostasis in Arabidopsis thaliana. Plant Physiol. 2016; 170:2478-2493.

27. Yi K, Wu Z, Zhou J, Du L, Guo L, Wu Y, Wu P. OsPTF1, a novel transcription factor involved in tolerance to phosphate starvation in rice. Plant Physiol. 2005; 138, 2087-2096.

28. Yang T, Hao L, Yao S, Zhao Y, Lu W, Xiao K. TabHLH1, a bHLH-type transcription factor gene in wheat, improves plant tolerance to $\mathrm{Pi}$ and $\mathrm{N}$ deprivation via regulation of nutrient transporter gene transcription and ROS homeostasis. Plant Physiol Bioch. 2016;104:99-113.

29. Yang L, Hu H, Zhu B, Jin X, Wu F, Zhang G. Genotypic variations of N use efficiency in Tibetan wild and cultivated barleys. J Zhejiang Univ (Agric \& Life Sci). 2014; 40:155-64.

30. Quan X, Zeng J, Han Z, Zhang G. lonomic and physiological responses to low nitrogen stress in Tibetan wild and cultivated barley. Plant Physiol Bioch. 2017; 111:257-65.

31. Kang WH, Kim S, Lee HA, Choi D, Yeom SI. Genome-wide analysis of Dof transcription factors reveals functional characteristics during development and response to biotic stresses in pepper. Sci Rep. 2016; 6:33332

32. Chen $\mathrm{C}$, Chen $\mathrm{H}$, Zhang $\mathrm{Y}$, Thomas HR., Frank MH, He Y, Xia R. TBtools: an integrative toolkit developed for interactive analyses of big biological data. Mol Plant. 2020; 13: 1194-1202.

33. The International Barley Genome Sequencing Consortium. A physical, genetic and functional sequence assembly of the barley genome. Nature. 2012; 491:711-716.

34. Li F, Guo S, Zhao Y, Chen D, Chong K, Xu Y. (2010). Overexpression of a homopeptide repeatcontaining bHLH protein gene (OrbHLH001) from Dongxiang Wild Rice confers freezing and salt tolerance in transgenic Arabidopsis. Plant Cell Rep. 2010;29:977-986.

35. Xu W, Zhang N, Jiao Y, Li R, Xiao D, Wang Z. The grapevine basic helix-loop-helix (bHLH) transcription factor positively modulates CBF-pathway and confers tolerance to cold-stress in Arabidopsis. Mol Biol Rep. 2014;41:5329-5342.

36. Wang HY, Klatte M, Jakoby M, Bäumlein H, Weisshaar B, Bauer P. Iron deficiency-mediated stress regulation of four subgroup Ib BHLH genes in Arabidopsis thaliana. Planta. 2007;226:897-908.

37. Kent WJ, Baertsch R, Hinrichs A, Miller W, Haussler D. Evolution's cauldron: duplication, deletion, and rearrangement in the mouse and human genomes. Proc Natl Acad Sci USA. 2003;100:11484-11489.

38. Cannon SB, Mitra A, Baumgarten A, Young ND, May G. The roles of segmental and tandem gene duplication in the evolution of large gene families in Arabidopsis thaliana. BMC Plant Biol. 2004;4:10.

39. Flagel LE, Wendel JF. Gene duplication and evolutionary novelty in plants. New Phytol. 2009;183:557-64.

40. Ogo Y, Nakanishi IR, Nakanishi H, Kobayashi T, Takahashi M, Mori S, Nishizawa NK. The rice bHLH protein OsIRO2 is an essential regulator of the genes involved in Fe uptake under Fe-deficient 
conditions. Plant J. 2007;51:366-377.

41. Lea US, Slimestad R, Smedvig P, Lillo C. Nitrogen deficiency enhances expression of specific MYB and bHLH transcription factors and accumulation of end products in the flavonoid pathway. Planta. 2007;225:1245-1253.

42. Feyissa DN, Lovdal T, Olsen KM, Slimestad R, Lillo C. The endogenous GL3, but not EGL3, gene is necessary for anthocyanin accumulation as induced by nitrogen depletion in Arabidopsis rosette stage leaves. Planta. 2009;230:747-754.

43. He Z, Li L, Luan S. Immunophilins and parvulins. Superfamily of peptidyl prolyl isomerases in Arabidopsis. Plant Physiol. 2004;134:1248-1267.

44. Song Z, Pan F, Yang C, Jia H, Jiang H, He F, Li N, Lu X, Zhang H. Genome-wide identification and expression analysis of HSP90 gene family in Nicotiana tabacum. BMC Genet. 2019;20:35.

45. Eddy SR. Accelerated profile HMM searches. PLoS Comput Biol. 2011;7:e1002195.

46. Finn RD, Coggill P, Eberhardt RY, Eddy SR, Mistry J, Mitchell AL, Potter SC, Punta M, Qureshi M, Sangradorvegas A. The Pfam protein families database: towards a more sustainable future. Nucleic Acids Res. 2016;44:D279.

47. Marchler-Bauer A, Derbyshire MK, Gonzales NR, Lu S, Chitsaz F, Geer LY, et al. CDD: NCBI's conserved domain database. Nucleic Acids Res. 2015;43:222-2266.

48. Artimo P, Jonnalagedda M, Arnold K, Baratin D, Csardi G, Castro ED, Flegel V, Fortier A, Gasteiger E, Ioannidis V. ExPASy: SIB bioinformatics resource portal. Nucleic Acids Res. 2012;40:597-603.

49. Thompson JD, Higgins DG, Gibson TJ. CLUSTAL W: improving the sensitivity of progressive multiple sequence alignment through sequence weighting, position-specific gap penalties and weight matrix choice. Nucleic Acids Res. 1994;22:4673-4680.

50. Bailey TL, Boden M, Buske FA, Frith M, Grant CE, Clementi L, Ren J, Li WW, Noble WS. MEME Suite: tools for motif discovery and searching. Nucleic Acids Res. 2009; 37:202-208.

51. Chao J, Kong Y, Wang Q, Sun Y, Gong D, Lv J, Liu G. Mapgene2chrom, a tool to draw gene physical map based on perl and svg languages. Hereditas. 2015;37:91-97.

52. Wang Y, Tang H, Debarry JD, Tan X, Li J, Wang X, Lee T, Jin H, Marler B, Guo H. MCScanX: a toolkit for detection and evolutionary analysis of gene synteny and collinearity. Nucleic Acids Res. 2012;40:e49.

53. Krzywinski M, Schein Jl. Circos: an information aesthetic for comparative genomics. Genome Res. 2009;19:1639-1645.

54. Lescot M, Déhais P, Thijs G, Marchal K, Moreau Y, Yves VDP, Pieree R, Stephane R. Plantcare, a database of plant cis-acting regulatory elements and a portal to tools for in silico analysis of promoter sequences. Nucleic Acids Res. 2002;30:325-327.

55. Quan X, Zeng J, Ye L, Chen G, Han Z, Shah JM, Zhang G. Transcriptome profiling analysis for two Tibetan wild barley genotypes in responses to low nitrogen. BMC Plant Biol. 2016;16:30. 
56. Trapnell C, Williams BA, Pertea G, Mortazavi A, Kwan G, Van Baren MJ, et al. Transcript assembly and quantification by RNA-Seq reveals unannotated transcripts and isoform switching during cell differentiation. Nat biotechnol. 2010;28:511-515.

57. Robinson MD, McCarthy DJ, Smyth GK. edgeR: a Bioconductor package for differential expression analysis of digital gene expression data. Bioinformatics. 2010;26:139-40.

58. Schmittgen TD, Livak KJ. Analyzing real-time PCR data by the comparative CT method. Nat Protoc. 2008;3:1101-1108.

\section{Supplementary Information}

Additional file 1: Table S1 Characteristics of bHLH family genes in barley

Additional file 2: Fig. $\mathbf{S 1}$ Conserved motifs of bHLH domains in barley

Additional file 3: Table S2 Gene ID and sequences of $168 \mathrm{HvbHLH}$ identified in this study

Additional file 4: Table S3 Duplication genes among $H v b H L H$ genes

Additional file 5: Table S4 Predicted cis-elements in HvbHLH promoters

Additional file 6: Table S5 The expression profiles (FPKM) of the HvbHLH in barley cultivar 'Morex' under different tissues

Additional file 7: Table 66 Gene accession numbers and sequences of 16 HvbHLHDEGs in XZ149 and XZ56 under LN stress

Additional file 8: Table S7 The FPKM value of 16 HvbHLHDEGs in XZ149 and XZ56

Additional file 9: Table S8 DEGs at $6 \mathrm{~h}$ and $48 \mathrm{~h}$ after low $\mathrm{N}$ stress in XZ149 and XZ56

Additional file 10: Fig. S2 Real time PCR validation of 6 bHLH DEGs. a Transcript levels of 6 DEGs and the corresponding expression data of RNA-SEq. The bars represent SE $(n=3)$. The columns represent relative expression obtained by RNA-Seq, and solid lines represent relative expression obtained by qRT-PCR. $b$ Comparison between the relative expression obtained from RNA-Seq data and qRT-PCR. The RNA-Seq value of the relative expression ( $y$-axis) has been plotted against the developmental stages ( $x$-axis).

Additional file 11: Fig. S3 Expression analysis of the $H v b H L H$ genes in wild barley XZ149 and XZ56 at 8 time points after $L N$ stress. Expression data were the values of $L N / c o n t r o l$ at each time point. The color scale represents relative expression levels from high (red) to low (blue).

Additional file 12: Table S9 Gene ID and sequences of $b H L H$ used for search in Arabidopsis and rice Additional file 13: Table S10 Primers used in real time PCR 
Figures

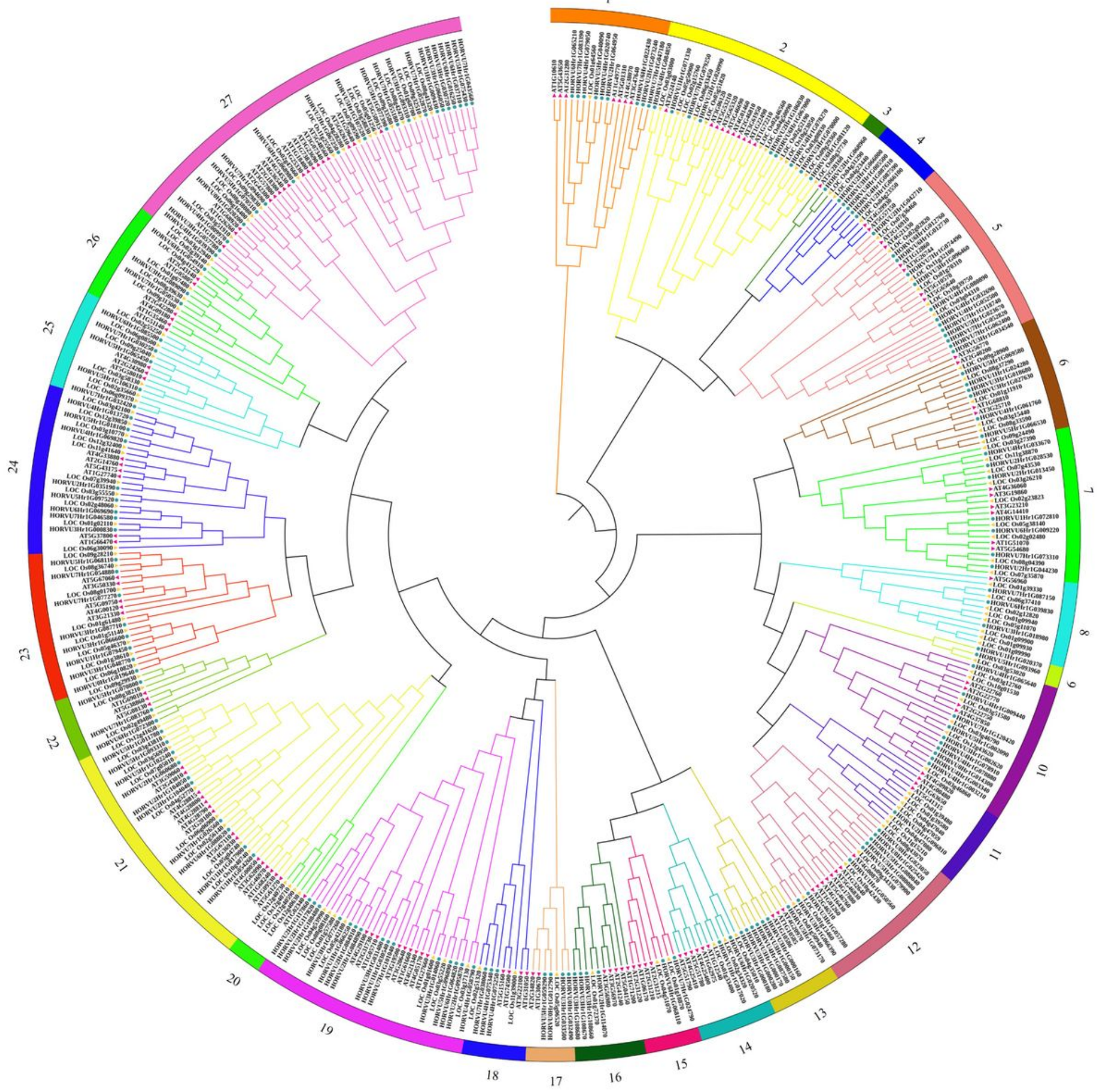

Figure 1

Phylogenetic tree of bHLH families in barley, Arabidopsis and rice. The different-colored arcs indicate different subgroups of the bHLHs. The unrooted neighbor-joining phylogenetic tree was constructed using MEGA6.0 with full-length amino acid sequences of $450 \mathrm{bHLHs}$, and the bootstrap test replicate was 
set as 1000 times. The circles, purple and yellow triangles represent the bHLH proteins from barley, Arabidopsis and rice, respectively.

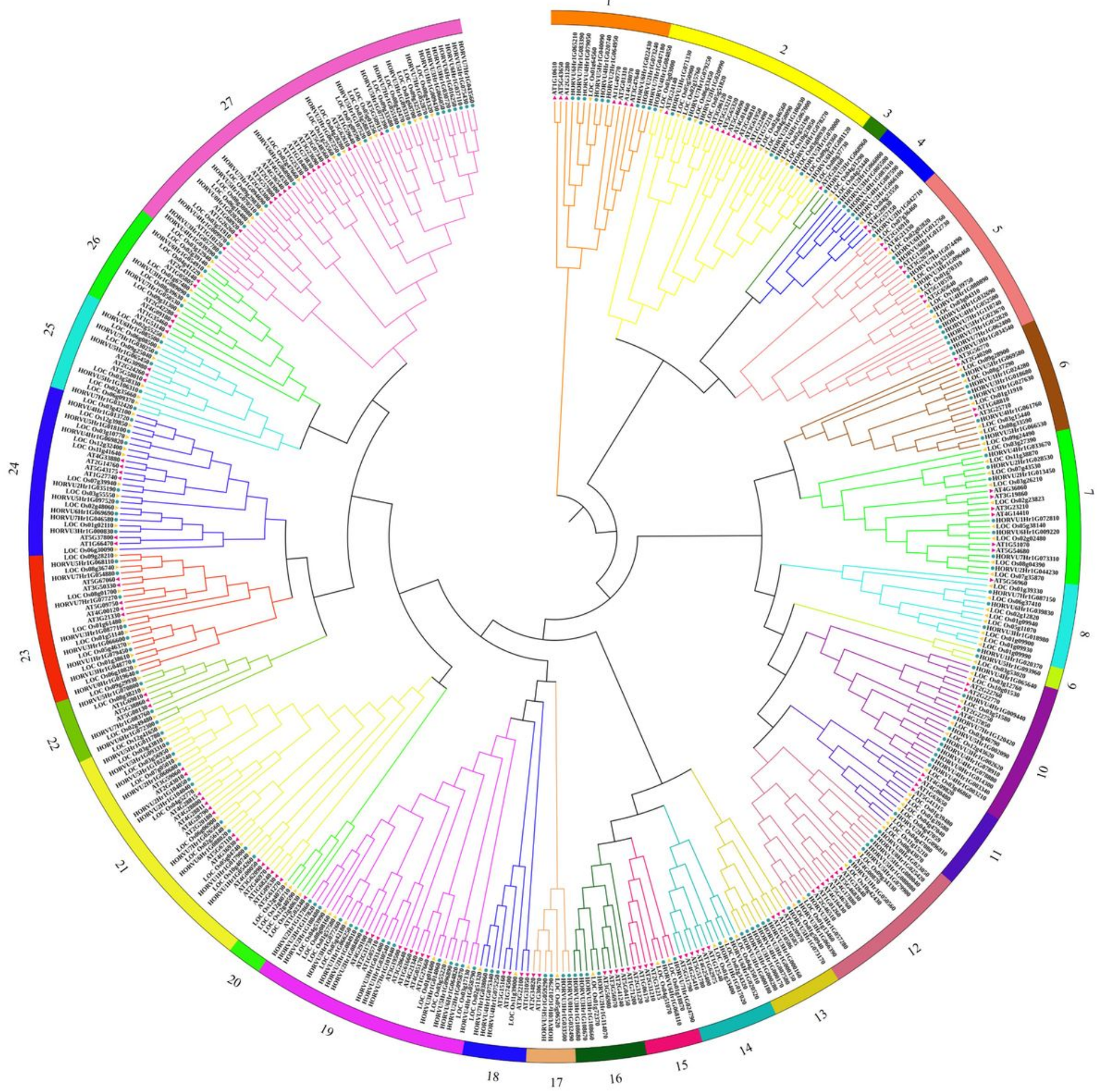

\section{Figure 1}

Phylogenetic tree of bHLH families in barley, Arabidopsis and rice. The different-colored arcs indicate different subgroups of the bHLHs. The unrooted neighbor-joining phylogenetic tree was constructed using MEGA6.0 with full-length amino acid sequences of $450 \mathrm{bHLHs}$, and the bootstrap test replicate was 
set as 1000 times. The circles, purple and yellow triangles represent the bHLH proteins from barley, Arabidopsis and rice, respectively.
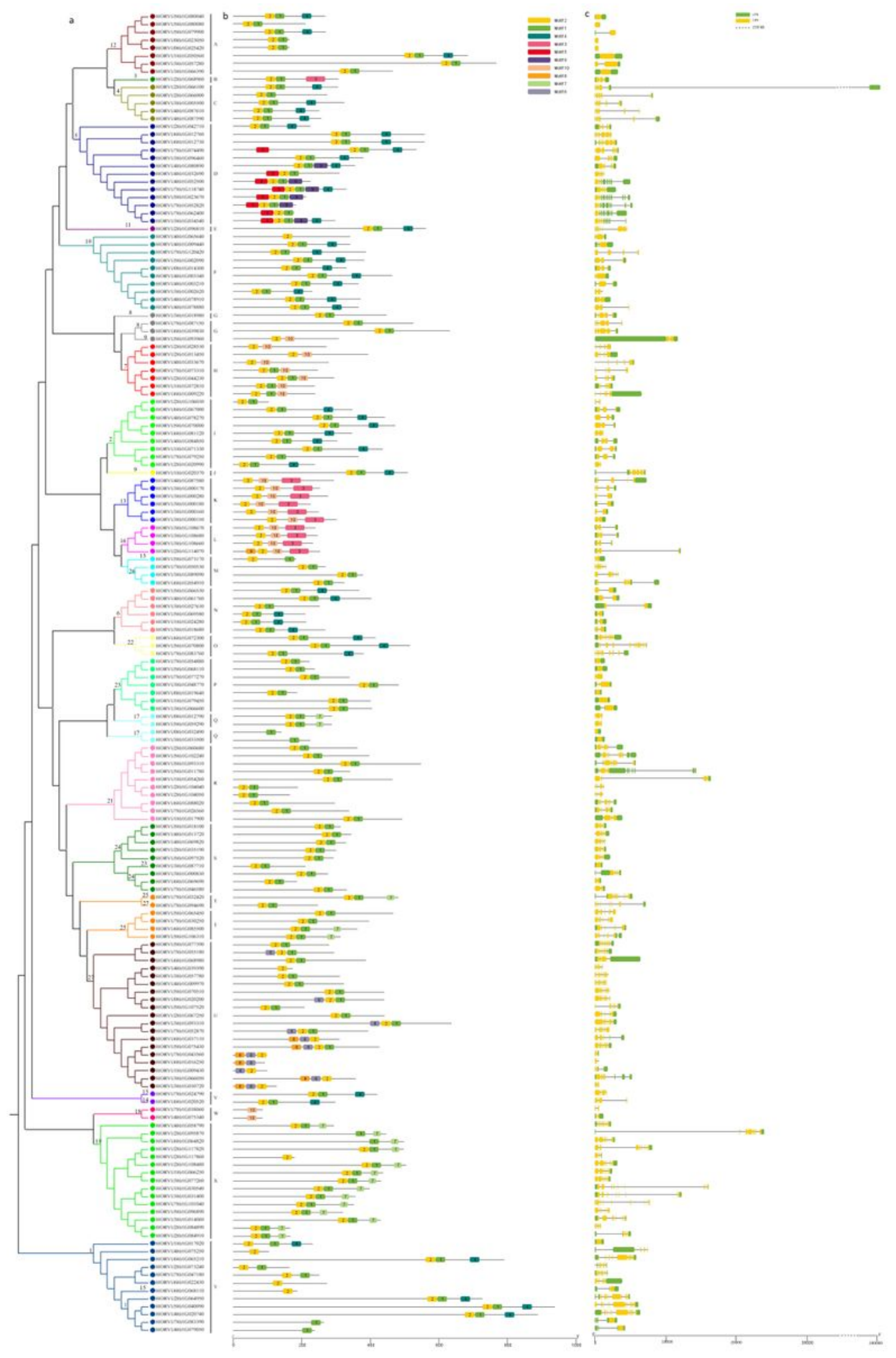

Figure 2

Phylogenetic relationships, conserved motifs and gene structure of the bHLH genes from barley. (a) Phylogenetic tree of $168 \mathrm{HvbHLH}$ proteins. The unrooted neighbor-joining phylogenetic tree was constructed with MEGA6.0 using full-length amino acid sequences of $168 \mathrm{HvbHLH}$ proteins, and the 
bootstrap test replicate was set as 1000 times. (b) The motif composition of HvbHLH proteins. The motifs, numbers 1-10, are displayed in different colored boxes. The sequence information for each motif is showed in Fig. S1. The length of protein can be estimated using the scale at the bottom. (c) Exon/intron structure of HvbHLH genes. Yellow boxes represent exons and black lines represent introns. The upstream/downstream region of HvbHLH genes are indicated in green boxes. The length of exons can be inferred by the scale at the bottom.
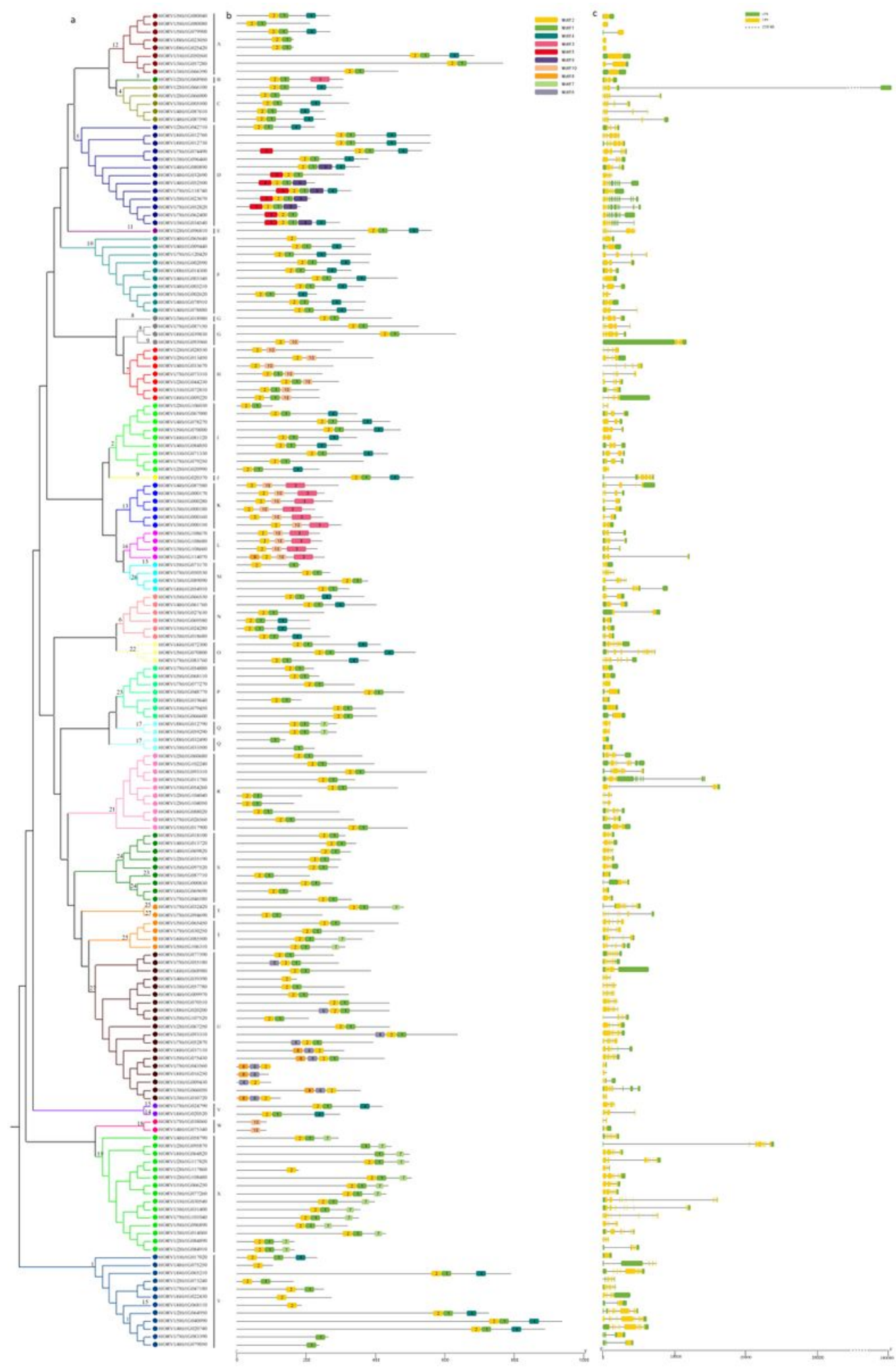

Figure 2 
Phylogenetic relationships, conserved motifs and gene structure of the bHLH genes from barley. (a) Phylogenetic tree of $168 \mathrm{HvbHLH}$ proteins. The unrooted neighbor-joining phylogenetic tree was constructed with MEGA6.0 using full-length amino acid sequences of $168 \mathrm{HvbHLH}$ proteins, and the bootstrap test replicate was set as 1000 times. (b) The motif composition of HvbHLH proteins. The motifs, numbers 1-10, are displayed in different colored boxes. The sequence information for each motif is showed in Fig. S1. The length of protein can be estimated using the scale at the bottom. (c) Exon/intron structure of HvbHLH genes. Yellow boxes represent exons and black lines represent introns. The upstream/downstream region of HvbHLH genes are indicated in green boxes. The length of exons can be inferred by the scale at the bottom.
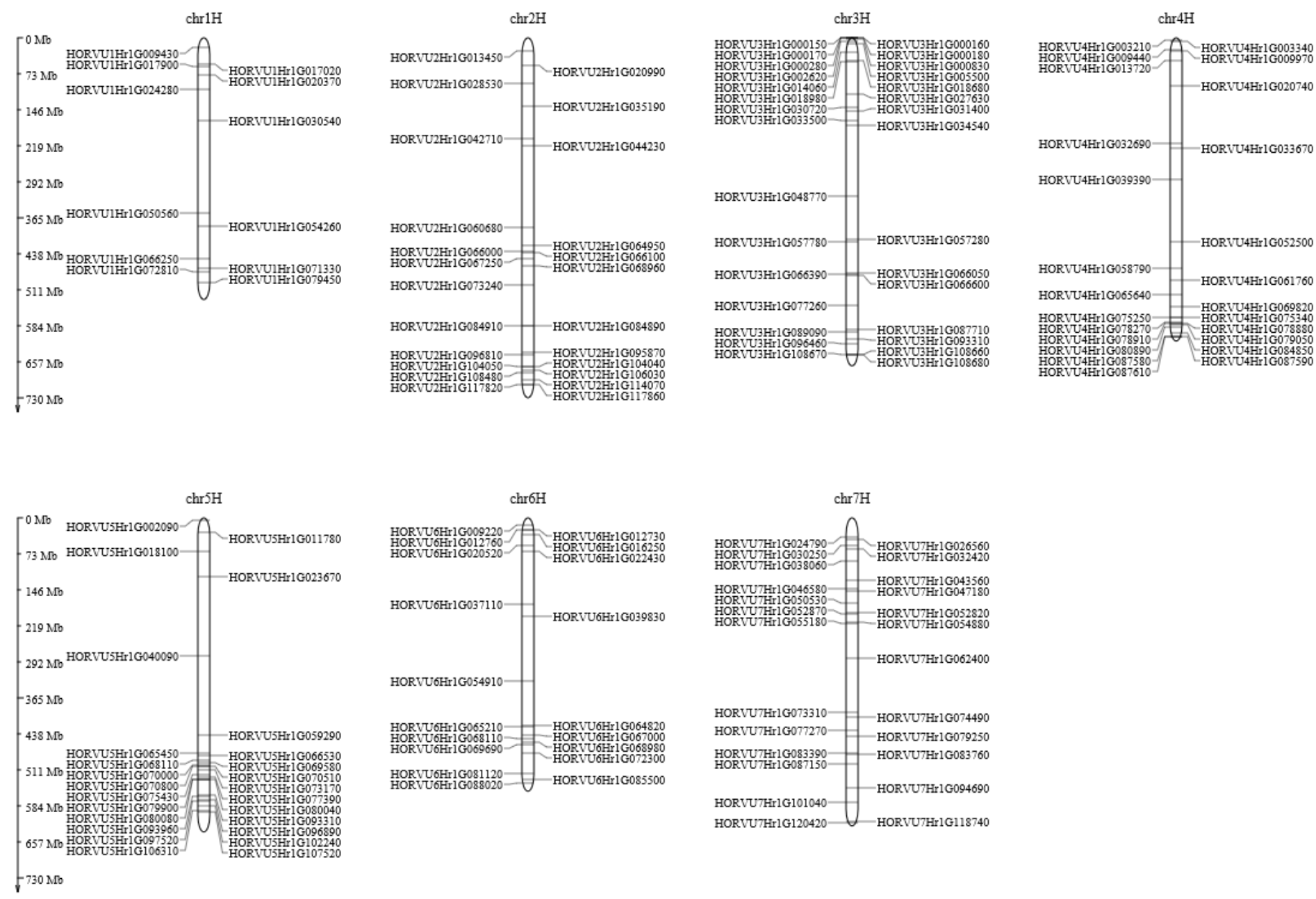

\section{Figure 3}

Chromosomal distribution of bHLH genes in barley. The chromosomes are numbered between 1 and 7 and shown at the top of each chromosome. Chromosomal distances are given in $\mathrm{Mb}$, and left number represent physical location on chromosomes of HvbHLHs. 

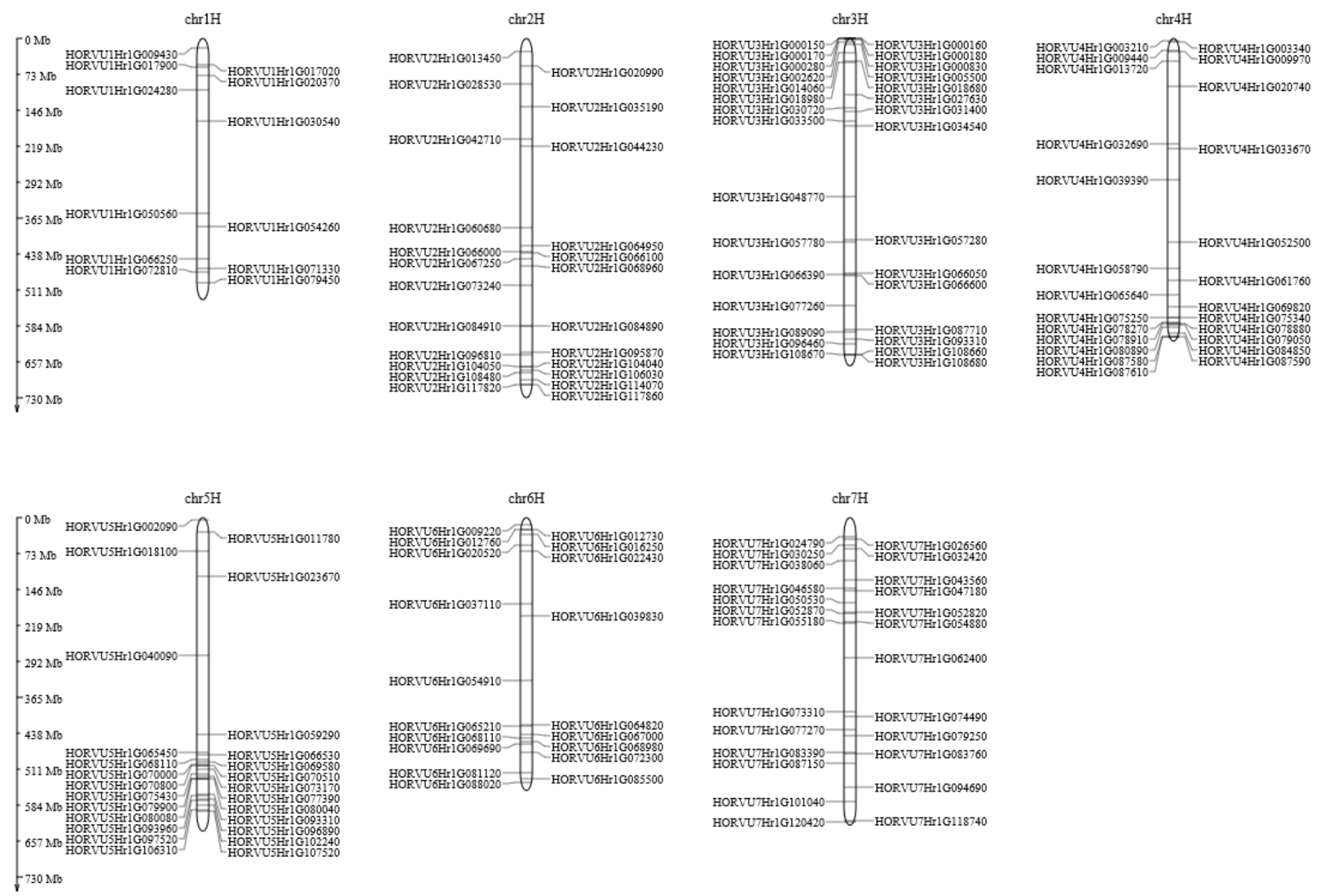

\section{Figure 3}

Chromosomal distribution of bHLH genes in barley. The chromosomes are numbered between 1 and 7 and shown at the top of each chromosome. Chromosomal distances are given in $\mathrm{Mb}$, and left number represent physical location on chromosomes of HvbHLHs. 


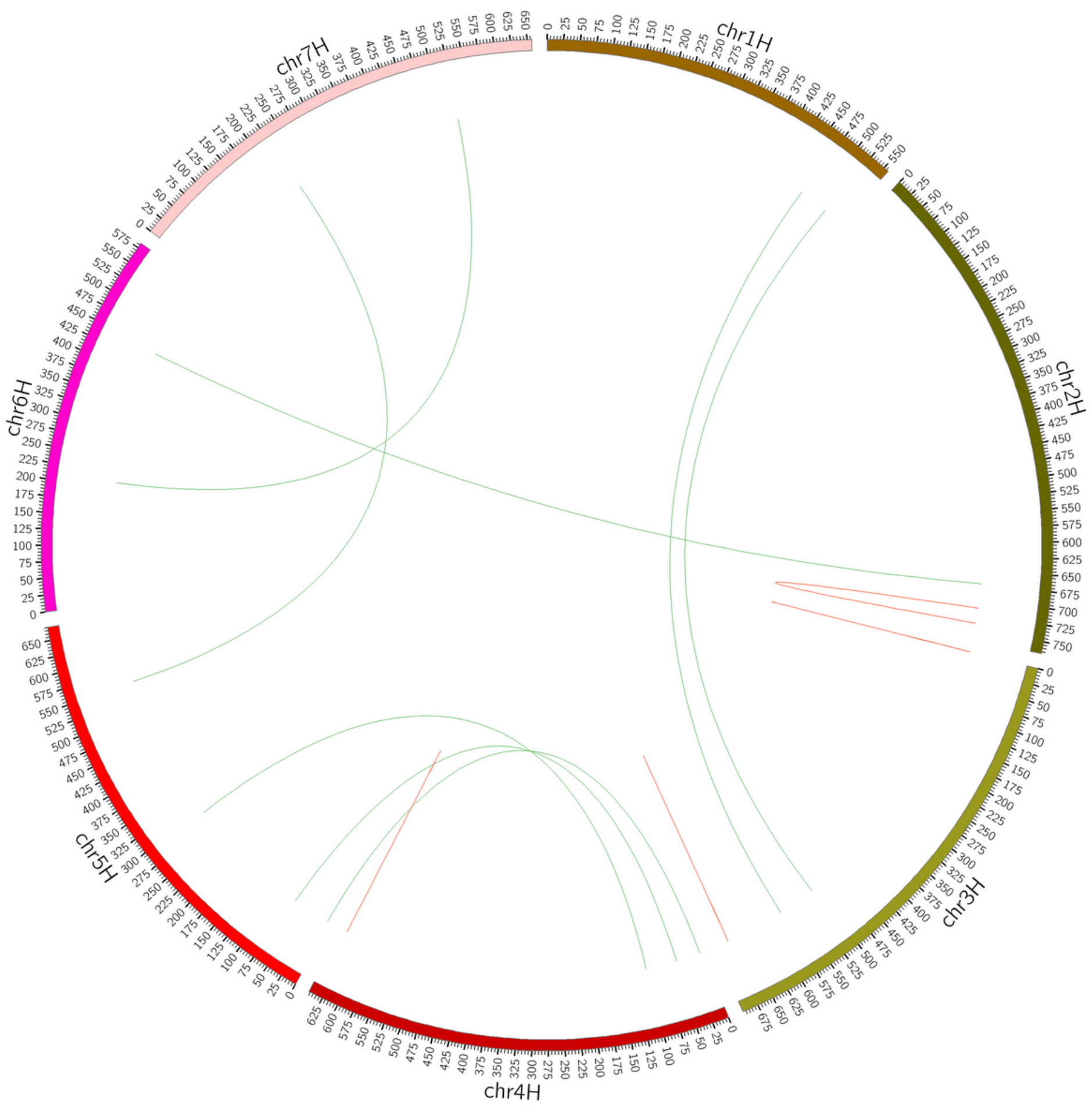

\section{Figure 4}

The synteny analysis of HvbHLH family in barley. Gray lines indicate all synteny blocks in the barley genome, and the red lines indicate duplicated HvbHLH gene pairs. The chromosome number is indicated at the bottom of each chromosome. 


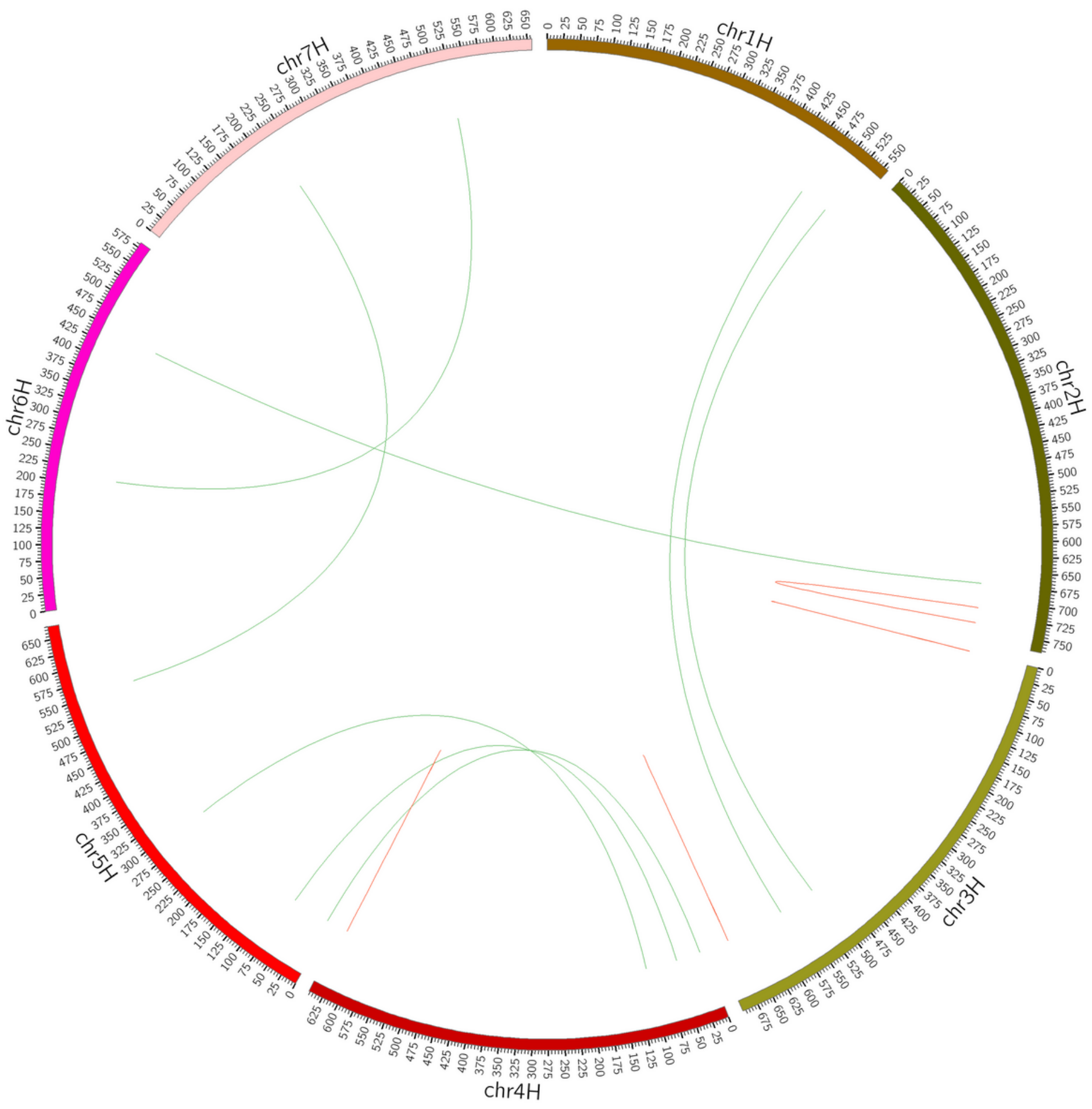

\section{Figure 4}

The synteny analysis of HvbHLH family in barley. Gray lines indicate all synteny blocks in the barley genome, and the red lines indicate duplicated HvbHLH gene pairs. The chromosome number is indicated at the bottom of each chromosome. 

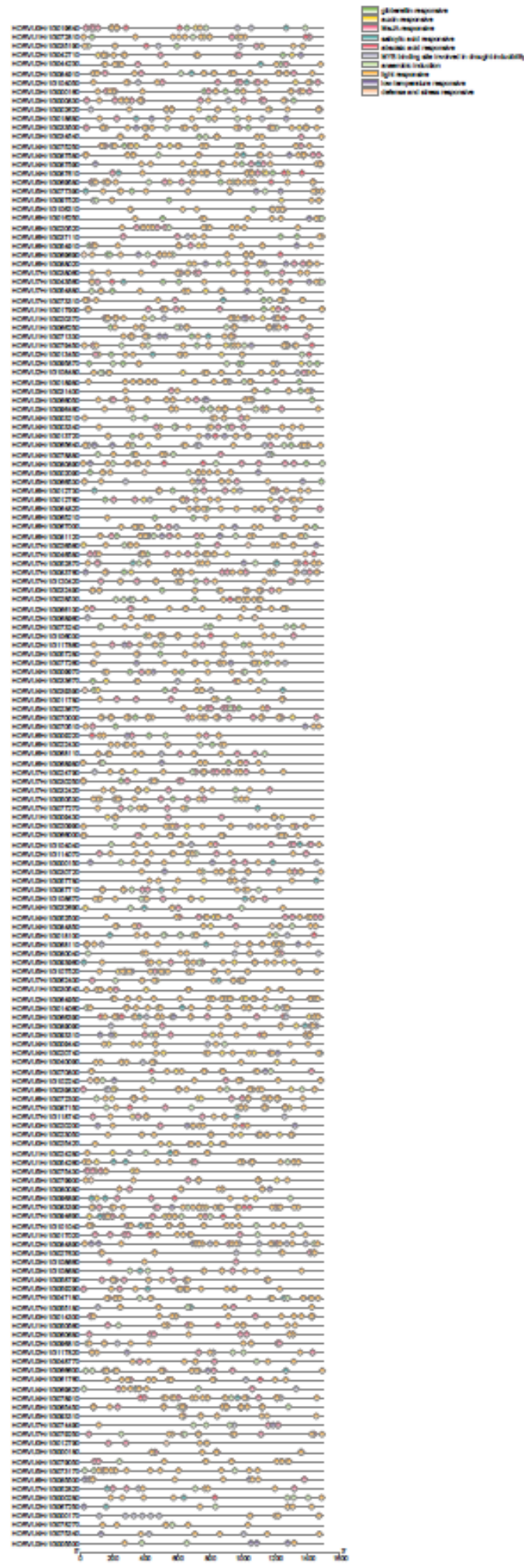

\section{Figure 5}

Predicted cis-elements in HvbHLH promoters. Promoter sequences (-1500 bp) of $168 \mathrm{HvbHLH}$ genes were analyzed by PlantCARE. The upstream length to the translation starting site can be inferred according to the scale at the bottom. 

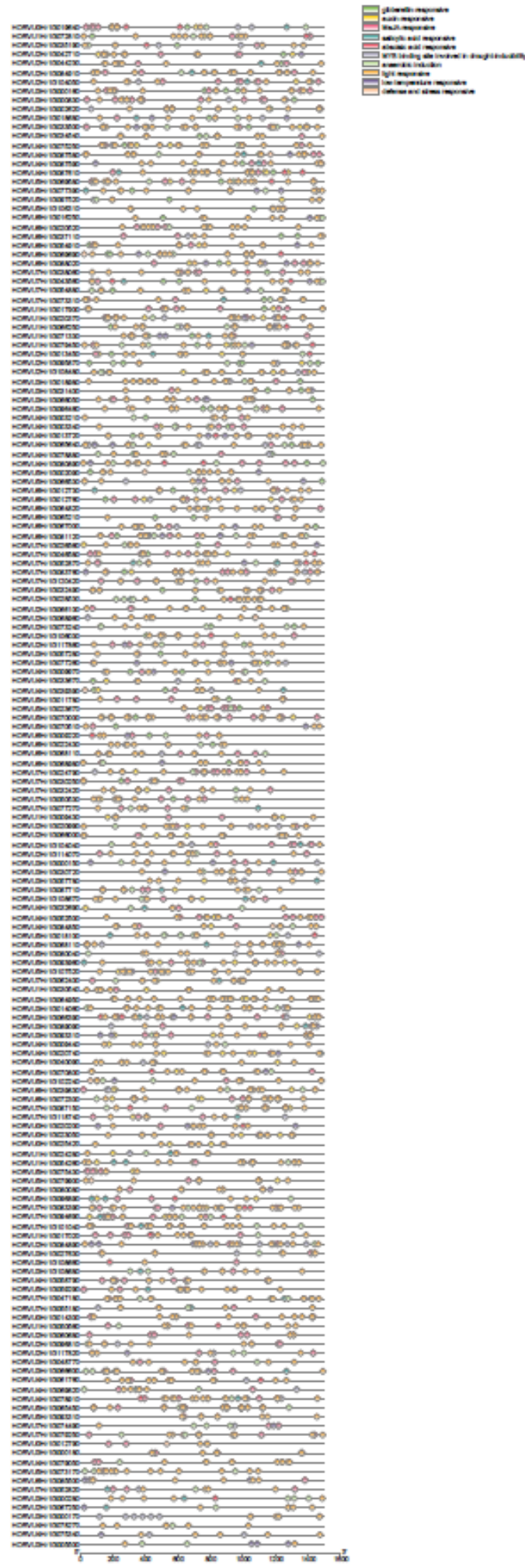

\section{Figure 5}

Predicted cis-elements in HvbHLH promoters. Promoter sequences (-1500 bp) of $168 \mathrm{HvbHLH}$ genes were analyzed by PlantCARE. The upstream length to the translation starting site can be inferred according to the scale at the bottom. 


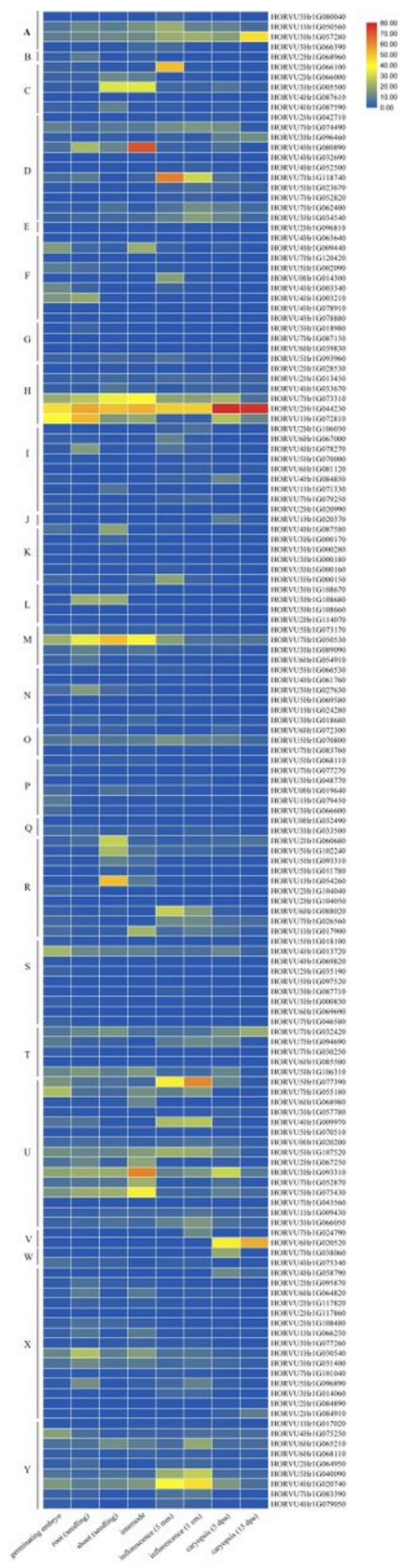

\section{Figure 6}

Expression profiles (FPKM) of the HvbHLH genes in different tissues of barley cultivar Morex. The color scale represents relative expression levels from high (red) to low (blue). 


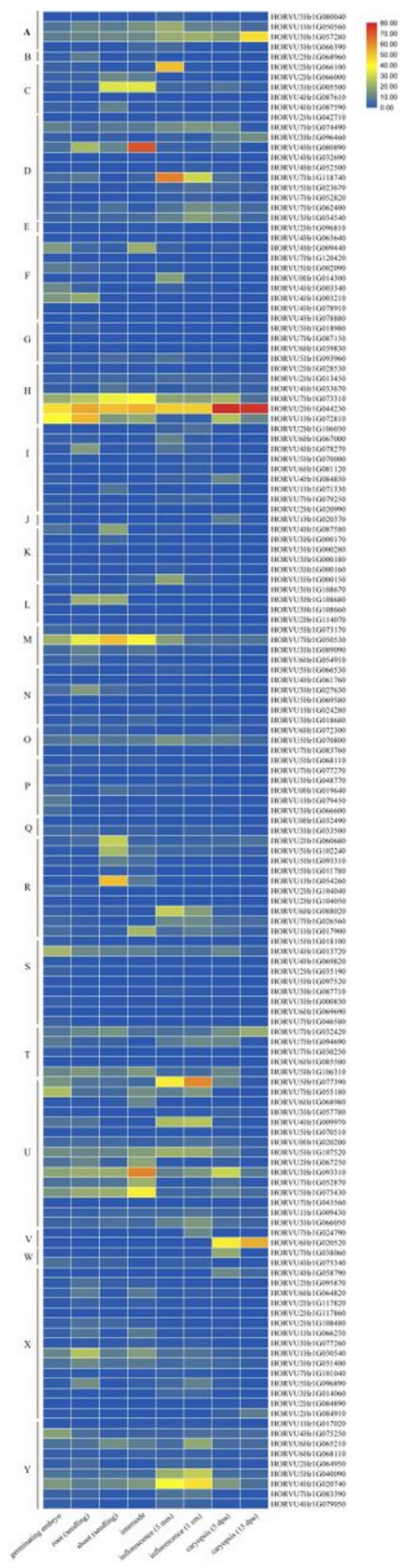

\section{Figure 6}

Expression profiles (FPKM) of the HvbHLH genes in different tissues of barley cultivar Morex. The color scale represents relative expression levels from high (red) to low (blue). 


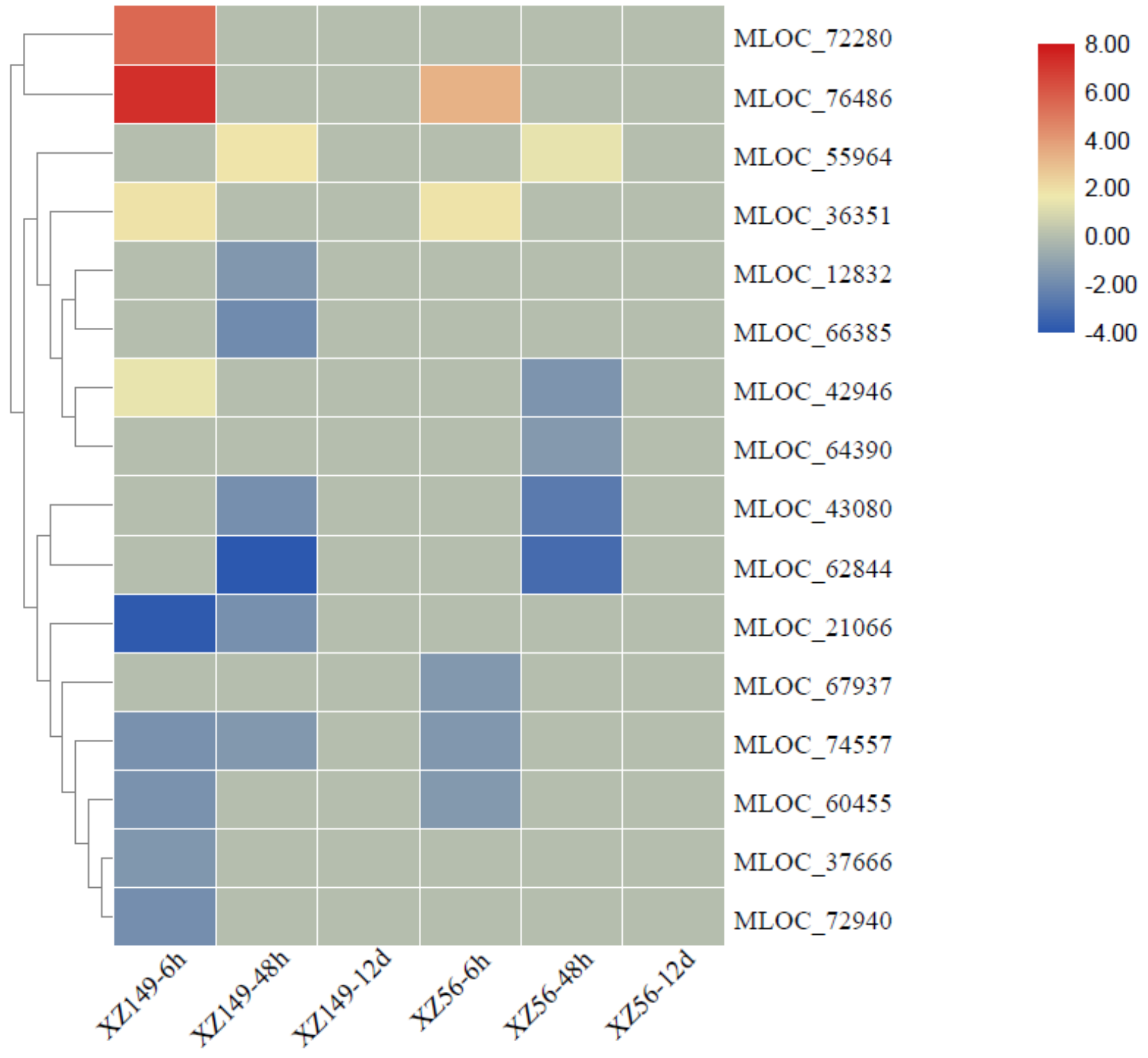

Figure 7

Expression profiles of the HvbHLH genes in wild barley XZ149 and XZ56 at $6 \mathrm{~h}, 48 \mathrm{~h}$, and $12 \mathrm{~d}$ under LN stress. Expression data were the values of $\mathrm{LN} /$ control at each time point. The color scale represents relative expression levels from high (red) to low (blue). 


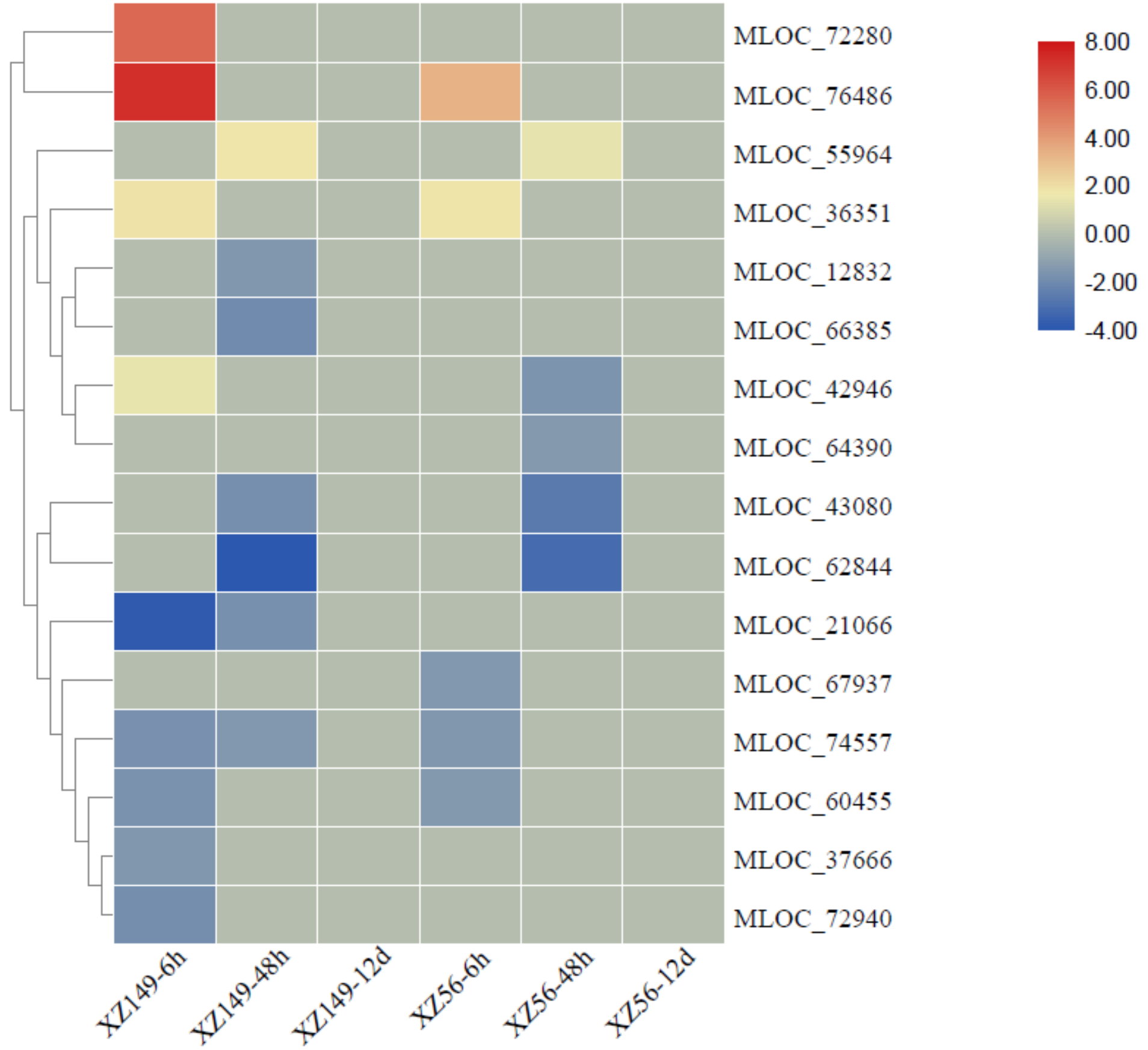

Figure 7

Expression profiles of the HvbHLH genes in wild barley XZ149 and XZ56 at $6 \mathrm{~h}, 48 \mathrm{~h}$, and $12 \mathrm{~d}$ under LN stress. Expression data were the values of $\mathrm{LN} /$ control at each time point. The color scale represents relative expression levels from high (red) to low (blue).

\section{Supplementary Files}


This is a list of supplementary files associated with this preprint. Click to download.

- Fig.S1.jpg

- Fig.S1.jpg

- Fig.S2.tif

- Fig.S2.tif

- Fig.S3.pdf

- Fig.S3.pdf

- TableS.xlsx

- TableS.xlsx 